Cite as: Stephane H Maes, (2020), "Superstrings Encounter of the Second, Third or Fourth Types?", viXra:2010.0140v1, https://vixra.org/pdf/2010.0140v1.pdf, https://shmaesphysics.wordpress.com/2020/07/19/ superstrings-encounter-of-the-second-third-or-fourth-types/, July 5, 2020.

\title{
Superstrings Encounters of the Second, Third or Fourth Types?
}

\author{
Stephane H. Maes ${ }^{1}$
}

July 12,2020

\begin{abstract}
:
Strings and their evolutions as superstrings and M-theory have been one of the main focus of theoretical Physics for the last 40 years. In fact some have characterized superstrings and $M$-theory as the best candidates at explaining quantum gravity and providing a Theory of Everything. Others have claimed that it is "Physics of the next century provided for our exploration this century". Yet not much has come out of it in terms of actual predictions or observation of anything about anything.

In the context of explaining gravity with entanglement with multi-fold mechanisms, we encountered traces of superstrings and may have met some of them or their impacts. It qualifies for an alien encounter of the third type. Dualities between spacetime and superstrings were derived, yet nobody embarked or will ever embark on a superstring spacetime trip, at least. So no encounter of the fourth type.

This paper summarizes what we have determined about strings, superstrings and M-theory as part of the multi-fold universe models. The observations and lessons learned are telling signs for superstring investigators.
\end{abstract}

\section{Introduction}

Strings and their evolutions as superstrings and M-theory have been one of the main focus of theoretical Physics for the last 40 years. In fact some have characterized superstrings and $\mathrm{M}$-theory as the best candidates at explaining quantum gravity and provide a Theory of Everything (ToE). Others have claimed it is physics of the next century provided for our exploration this century. Yet not much has come out of it in terms of actual predictions or any subsequent observations.

The present paper characterizes the properties of strings, superstrings and M-Theory as we encountered unexpected hints of them as part of the multi-fold universe proposal [1], that proposes to address the Einstein Podolsky Rosen (EPR) paradox and encountered massless and massive gravity as a result, including recovery of General relativity (GR) at large scales, and a discrete, fractal, non-commutative, Lorentz invariance spacetime generated by random walks at very small scales. See [1] for more details.

In [2], we discussed the resulting lessons learned and dualities with respect to superstrings and super symmetry. [3] then expanded the analysis to Grand Unification Theories (GUTs) and Theories of Everything and [4] did the same for Loop Quantum Gravity, the main quantum gravity model that competes with Superstrings.

The present paper aims solely at capturing in one place, how strings, superstrings and M-theory (which we will now simplify designate as superstrings) relate to our spacetime, at least in the context of [1]. It should be of interest to all those who have interest in superstrings. As observations are (still) non-existent, theoretical chance

\footnotetext{
${ }^{1}$ shmaes.physics@gmail.com
} 
encounters are worth keeping track of, just like UFOs... See [5] for the UFO and Alien encounter classification analogy we relied upon here.

In this paper, we remain at a high level of discussion. It makes the points accessible to a wider audience and keeps the door open to further papers or discussions devoted to details of interest. Yet it requires the reader to review $[1,2]$, as we do not revisit here all the details of the multi-fold mechanism or reconstruction of spacetime. This paper is rather a story tale. "[1]" appears often, as a person or a model, to refer to the original arguments, analysis, mechanisms or proposals discussed in [1].

Note on 10/17/20: Work on multi-fold universes is continuing [19]. More recent result have further advanced our understanding of superstrings in multi-fold universes as well as in the real universe [20-22]. We strongly recommend reading of these references, in their order of citation, after reviewing the present paper.

\section{Second, Third or Fourth type encounters of Superstrings?}

The claim of fame of strings came when it was observed that gravitons, or at least spin 2 massless strings/particles, emerge from the theory and that it models well GR perturbative models up to high order apparently without divergences. See [6] for some history. To this date, these points are presented as the most resounding validation of superstrings (as gravitons would not have been intended but emerged from the model) and the motivation for quasi cult-like belief that superstring will lead to the final ToE. Unfortunately for superstrings, and contrary to conventional statements, these results were actually baked in from the beginning when the Physics of strings with action that extremize the area between initial and final position (just like for soap membranes or bubbles) $[2,7,9]$ : it's the same action as the Hilbert Einstein action, at least to the first order! So their appearance is at best the result of trying to model quarks in mesons as strings according to the dual resonance model and such that confinement is modeled by stretched strings that when broken results into two mesons (i.e. create its own new quark / anti quark pair rather than let the quarks live alone). Gravitons are one type of strings (closed bosonic per the theory).

Yet, superstring theory has already determined that the theory can not exist stable in a universe with positive cosmological constant [12]. It can only exist in a negative curvature universe or a flat universe. We do know that our universe rather appears to have a positive curvature and a positive cosmological constant (and we know matter can't exist in an AdS spacetime in a stable manner [18]). The multi-fold mechanisms that recovers GR, can only account for generation of positive effective curvature and hence a positively curved universe unless if the initial conditions (background spacetime) were negatively curved [1]. It explains the restrictions on where superstrings can exist!

So, gravitons cannot exist in our spacetime! This is aligned with [1], where the multi-folds are identified as equivalent to the gravitons and exist in $\operatorname{AdS}(5)$ (+ whatever additional dimensions, it does not matter, for this discussion) and have an effect in our spacetime via the multi-fold mechanisms and mappings discussed in [1]. So no encounter of the third type with gravitons nor any other superstrings. The only possible encounters of the second type are through the effective attractive potentials and curvatures associated to gravitons.

Also, as discussed in the spacetime reconstruction model of [1], spacetime matches multi-fold quantas. So, we are not in a spacetime built on superstrings: no fourth type encounter! Indeed, even in the context of M-theory and Dbranes, they cannot be associated positive cosmological constant; at best fine-tuned flat branes [17]. D-brane can exist on a positive curvature (again as initial condition0 but the same considerations as above apply! No Encounter of the fourth type is possible (we are not on a superstring spaceship... Although some future applications envisaged in [1] could lead to some counter argument; and yes multi-fold are traversed by path integrals. These was not what we meant by encounter of the fourth type. We really meant that we are not in a superstring spacetime (i.e. built by superstrings or consisting of a D-brane etc.). 
The multi-folds mechanism and mappings concretize the AdS/CFT correspondence conjecture $[13,16]$ as a fact: $\operatorname{AdS}(5)$ is tangent to any point of spacetime (or conversely). Mappings amount to the holographic effects between gravitons in AdS(5) (+ other dimensions) and our spacetime is responsible for gravity. It is not a conjecture; it is a concrete effect implemented via multi-folds.

The multi-folds themselves concretize in a possibly richer manner (i.e. without limitation a priori to multi-fold dynamics dictated by Hilbert Einstein Actions) the ER=EPR conjecture [14,15]: multi-folds are activated between EPR entangled particles and generate attractive effective potentials; something missed by the ER=EPR work so far.

These effects qualify as encounters of the second type. Besides the irony of the analogy, it is important; in multifold universes, without any a priori setup to model or meet strings or any prescription of an Hilbert Einstein derived action, we encounter feature reminiscent and consistent with superstrings (gravitons) outside our spacetime (and we recover GR) [1,2].

Superstring is replete of other strings besides the spin two closed bosonic string associated to gravitons. These other strings are supposed to be associated to other particles, in theory the particles we know and their super partners. Yet none have ever been observed and $[2,3,10,11]$ argues that such super partners, and the associated supersymmetry effects, may not exist in the universe unless if the ideas suggested in [2,3] proved sensible. But that would require additional holographic mapping mechanisms and their physical justification, i.e. not just postulating the existence of such a mechanism (as was done so far, until [1,2], for the AdS/CFT correspondence conjecture and the Holographic principle for gravity) but also justifying why it should be there and what it would entail.

\section{What about other alleged encounters of the third type?}

\subsection{Superstrings or Point Particles}

String and Superstring theories also argue particles appearance as strings instead of point particles (and wiggling with properties that would characterize the particles). It is another conventional explanation of how strings relate to our world.

In our view, not limited to multi-fold universes, we explain this differently:

- In spacetime, point particles appear with uncertainty as a fuzzy ball oscillating around. In the presence of gravity, they wiggle preferably along geodesics (it is in fact one of the reason why gravity breaks supersymmetry and prevent magnetic monopoles [11]).

- As discussed in [8], one can see strings wiggling on the horizon of black holes. Looking carefully, it is just a particular case of the previous bullet where the impression results from the horizon fluctuations due to uncertainties and changes in the horizon position due to the absorption and emission of particles.

We claim therefore that these are not strings, only appearances of strings due to gravity and uncertainty. Moving on, nothing to see here.

\subsection{Superstrings attached to spacetime}

Another popular view is that superstrings attach to point particles in our spacetime and wiggle in their own universe in ways that again characterize the particles they attach to. It relates to D-branes, AdS/CFT correspondence conjecture and M-theory.

As it is outside our spacetime, if it were to exist, we can only observe the effects, i.e. the particle properties. These would be by definition only encounters of the second type. Note that in multi-fold universes, particles appear as 
surrounded by microscopic black holes. They could be the attachment point for superstrings. Concretized spacetime appear similarly and the same behavior could exist with respect to graviton strings.

These may be results or alternatives to holographic principles in order to justify effects of strings in our spacetime. They are at best encounters of the second type.

\section{Conclusions}

In [1], we have encountered possible effects or traces of superstrings. They live a universe tangent to spacetime with impact due to holographic effects associated to the multi-fold mechanisms. The duality that seems to exist between multi-fold mechanisms able to recover GR and predict multiple falsifiable phenomena [1,2], is intriguing enough that we believe it should be investigated by string theorists in their quest to relate superstring and the real universe.

Unfortunately, while it seems that we have encountered traces of superstrings, we have also established that we can never encounter them: they are not physical to our spacetime and do not live in our spacetime. Only their effect can be seen and it certainly raises fundamental questions that superstring, supergravity and M-theory may also want to consider. Combined with [3], supersymmetry and GUTs have also questions to answer or new models to consider.

Superstring live in a different space tangent to our spacetime. They have gravity effects on our spacetime if they implement the multi-fold mechanisms. They could characterize particles if they attach to the microscopic black hole surrounding particle and spacetime locations in multi-fold universe. Or they do not contribute this way, multifolds are not related to superstrings; they just also live in $\operatorname{AdS}(5)$. Then whatever happens in that space (and its additional dimension) is unphysical and irrelevant to our spacetime. Superstring may exist and be perfectly modeled, but why bother?

[1] shows also significant impact on the Standard Model, when we add gravity (especially the short scale massive contributions). It could contribute explanations to several famous open issues; without requiring the New Physics offered by superstrings and supersymmetry. An always updated set of such examples can be found in [19].

Needless to say that [1] brings a lot of food for thoughts to theoretical physics. Hopefully, it will not be dismissed because still in a too early stage. Instead we hope for efforts to validate or falsify our predictions, and, in the present case, understand its implications on superstrings. [2] was introduced to emphasize the amazing dualities between multi-fold universes and superstrings. The present paper tries to call a spade a spade and to show exactly what our analysis of multi-fold mechanisms implies for superstrings. Sure, we can argue that our universe may not be characterized as a multi-fold universe, hence the need to falsify its claims then. But what about all the dualities with superstrings [2]? So far multi-fold mechanisms provide amazingly concrete physical explanations.

And so, yes, we demonstrated that only superstring encounters of the second type are possible in multi-fold universe. We believe and conjecture that is also true in the real universe.

References: (most references come from popular science to make the discussion more approachable)

[1]: Stephane H. Maes, (2020) "Quantum Gravity Emergence from Entanglement in a Multi-Fold

Universe", viXra:2006.0088v1, https://vixra.org/pdf/2006.0088v1.pdf (June 9, 2020).

[2]: Stephane H Maes, (2020), "Dualities or Analogies between Superstrings and Multi-fold

Universe", viXra:2006.0178v1, https://shmaesphysics.wordpress.com/2020/06/14/dualities-or-analogies-betweensuperstrings-and-multi-fold-universes/, June 14, 2020. 
[3]: Stephane H Maes, (2020), "Ultimate Unification: Gravity-led Democracy vs. Uber-

Symmetries", viXra:2006.0211v1, https://shmaesphysics.wordpress.com/2020/06/16/ultimate-unification-gravityled-democracy-vs-uber-symmetries/, June 16, 2020.

[4]: Stephane H Maes, (2020), "Alignments and Gaps Between Multi-fold Universes And Loop Quantum

Gravity", viXra:2006.0229v1, https://shmaesphysics.wordpress.com/2020/06/19/multi-fold-universes-analysis-ofloop-quantum-gravity/, June 18, 2020.

[5]: https://en.wikipedia.org/wiki/Close encounter

[6]: https://en.wikipedia.org/wiki/History of string theory

[7]: Edouard B. Manoukian, (2016), "Quantum Field Theory II: Introductions to Quantum Gravity, Supersymmetry and String Theory", Springer

[8]: G. 't Hooft, (1990), "The Black Hole Interpretation of String Theory", Nuclear Physics B335 (1990) 138-154

[9]: Zwiebach, Barton (2003). "A First Course in String Theory". Cambridge University Press.

[10]: Stephane H Maes, (2020), "Gravity Induced Anomalies Smearing in Standard Model so that Protons May

Never Decay, Except in Black Holes", viXra:2006.0128v1,

https://shmaesphysics.wordpress.com/2020/06/12/protons-may-never-decay-except-in-black-holes/, June 13, 2020.

[11]: Stephane H Maes, (2020), "Gravity or Magnetic Monopoles? You Cannot Have Both!", viXra:2006.0190v1, https://shmaesphysics.wordpress.com/2020/06/15/gravity-or-magnetic-monopoles-you-cannot-have-both/ , June 15, 2020.

[12]: Georges Obied, Hirosi Ooguri, Lev Spodyneiko, Cumrun Vafa, (2018), "De Sitter Space and the Swampland", arXiv:1806.08362v3.

[13]: J. M. Maldacena, (1998), "The Large N Limit Of Superconformal Field Theories And Supergravity", Adv. Theor. Math. Phys. 2 (1998) 231.

[14]: Maldacena, Juan and Susskind, Leonard (2013). "Cool horizons for entangled black holes", Fortsch. Phys. 61

(9): 781-811. arXiv:1306.0533

[15]: Julian Sonner, (2013), "Holographic Schwinger Effect and the Geometry of Entanglement", arXiv:1307.6850v3

[16]: Makoto Natsuume, (2015), "AdS/CFT Duality User Guide (Lecture Notes in Physics)", Springer; 2015 edition

(April 2, 2015)

[17]: Katrin Becker, Melanie Becker, John H. Schwarz, (2007), "String Theory and M-Theory: A Modern

Introduction", Cambridge University Press

[18]: Georgios Moschidis, (2018), "A proof of the instability of AdS for the Einstein--massless Vlasov system", arXiv:1812.04268v1.

[19]: Stephane H. Maes, (2020), "Web Site Tracking all Publications around the Multi-fold universe" -Navigation page listing all papers, https://shmaesphysics.wordpress.com/shmaes-physics-site-navigation/.

[20]: Stephane H Maes, (2020), "Tracking Down The Standard Model With Gravity In Multi-Fold

Universes", https://shmaesphysics.wordpress.com/2020/08/30/tracking-down-the-standard-model-with-gravityin-multi-fold-universes/, August 20, 2020.

[21]: Stephane H Maes, (2020), "Renormalization and Asymptotic Safety of Gravity in a Multi-Fold Universe: More Tracking of the Standard Model at the Cost of Supersymmetries, GUTs and Superstrings",

https://shmaesphysics.wordpress.com/2020/09/19/renormalization-and-asymptotic-safety-of-gravity-in-a-multifold-universe-more-tracking-of-the-standard-model-at-the-cost-of-supersymmetries-guts-and-superstrings/, September 18, 2020.

[22]: Stephane H Maes, (2020), "Circular Arguments in String and Superstring Theory from a Multi-fold Universe Perspective", https://shmaesphysics.wordpress.com/2020/10/11/circular-arguments-in-string-and-superstringtheory-from-a-multi-fold-universe-perspective/ , October 5, 2020. 